\title{
Mapping of Quantitative Trait Loci for Blood Pressure and Cardiac Mass in the Rat by Genome Scanning of Recombinant Inbred Strains
}

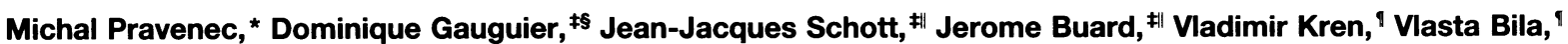 \\ Claude Szpirer, ${ }^{\star \star}$ Josiane Szpirer, ${ }^{\star \star}$ Jia-Ming Wang, \# Huaming Huang, \# Elizabeth St. Lezin, \# M. Anne Spence, ${ }^{\$ S}$

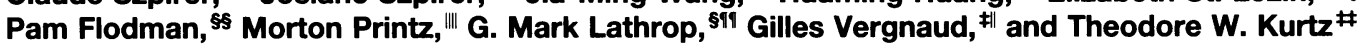 \\ *Institute of Physiology, Czech Academy of Sciences, Prague, Czech Republic; ${ }^{\ddagger}$ Laboratoire de Genetique Moleculaire, Centre d'Etudes \\ du Bouchet, Vert le Petit, France; ${ }^{\S}$ Wellcome Trust Centre for Human Genetics, Oxford, United Kingdom; "Laboratoire de Genetique des \\ Especes, Institut de Biologie, Nantes, France; 'Department of Biology, 1st Medical Faculty, Charles University, Prague, Czech Republic; \\ **Department of Molecular Biology, University Libre de Bruxelles, Rhode-St-Genese Belgium; ${ }^{\ddagger}$ Department of Laboratory Medicine, \\ University of California, San Francisco, California 94143; ${ }^{\$ \S}$ Department of Pediatrics, University of California, Irvine, California \\ 92613; "lil Department of Pharmacology, University of California, San Diego, California 92093; and "19 INSERM U358, Hopital St. Louis, \\ Paris, France
}

\begin{abstract}
In the HXB and BXH recombinant inbred strains derived from the spontaneously hypertensive rat and the normotensive Brown Norway rat, we determined the strain distribution patterns of 500 genetic markers to scan the rodent genome for quantitative trait loci regulating cardiac mass and blood pressure. The markers spanned $\sim 1,139 \mathrm{cM}$ of the genome and were tested for correlations with left ventricular mass adjusted for body weight, and with systolic, diastolic, and mean arterial pressures. The marker for the dopamine $1 \mathrm{~A}$ receptor $(\mathrm{Drdla})$ on chromosome 17 showed the strongest correlation with left ventricular heart weight $(P=$ $.00038, r=-0.59)$ and the relationship to heart weight was independent of blood pressure. The markers showing the strongest correlations with systolic, diastolic, and mean arterial pressure were $D 19$ Mit7 on chromosome $19(P=.0012$, $r=.55), D 2 N 35$ on chromosome $2(P=.0008, r=.56)$, and $I l 6$ on chromosome $4(P=.0018, r=.53)$, respectively. These studies demonstrate that the HXB and BXH strains can be effectively used for genome scanning studies of complex traits and have revealed several chromosome regions that may be involved in the genetic control of blood pressure and cardiac mass in the rat. (J. Clin. Invest. 1995. 96:19731978.) Key words: rat $\bullet$ genetics $\bullet$ blood pressure $\bullet$ hypertension - chromosome
\end{abstract}

\section{Introduction}

Linkage analysis of $\mathrm{F} 2$ and backcross populations is a powerful technique for the preliminary mapping of quantitative trait loci (QTLs) ${ }^{1}$ influencing complex phenotypes (1). However, this

Address correspondence to Michal Pravenec, PhD, Institute of Physiology, Czech Academy of Sciences, 1083 Videnska, Prague 14220 Czech Republic. Phone: 422-475-2297; FAX: 422-471-9517; E-mail: pravenec@biomed.cas.cz

Received for publication 7 April 1995 and accepted in revised form 12 June 1995.

1. Abbreviations used in this paper: BN, Brown Norway; QTL, quantitative trait loci; RI, recombinant inbred; SHR, spontaneously hypertensive rat.

J. Clin. Invest.

(C) The American Society for Clinical Investigation, Inc.

0021-9738/95/10/1973/06 \$2.00

Volume 96, October 1995, 1973-1978 approach has not seen widespread use in part because of the considerable amount of time and effort required to genotype and phenotype large numbers of animals in segregating populations. Recombinant inbred (RI) strains can be very useful for gene mapping (2-5) and may offer certain advantages over classical crosses for genome scanning and mechanistic studies of complex quantitative traits such as blood pressure or addictive behaviors (6-10). For example, in a given set of RI strains, the strain distribution patterns of multiple genetic markers need only be determined once and therefore, investigators can concentrate on phenotyping without having to run the thousands of genotyping reactions typically required in studies of large segregating populations. Large sets of extensively genotyped RI strains are available in the mouse and have been used to map QTLs influencing traits such as behavior and seizure susceptibility $(7,8,11,12)$. However, it is difficult to phenotype small animals for certain physiologic traits and as a consequence, mouse RI strains cannot be readily used to study complex phenotypes such as blood pressure or cardiac function.

The HXB and BXH strains represent the largest set of rat RI strains in the world and were initially derived from the spontaneously hypertensive rat (SHR) and the normotensive Brown-Norway rat for genetic studies of hypertension (10). These strains provide unique opportunities for mapping QTLs in the rat and could greatly expand the use of combined genetic and physiologic approaches to the study of a wide range of complex phenotypes, particularly those relevant to cardiovascular and renal physiology. In the current studies, we used the HXB and BXH RI strains to search for QTLs regulating blood pressure and cardiac mass in the SHR. Specifically, we determined the RI strain distribution patterns of 500 genetic markers and tested these markers for correlations with left ventricular mass and systolic, diastolic, and mean arterial pressure. In accord with preliminary studies in F2 populations derived from the spontaneously hypertensive rat, we have found evidence of blood pressure QTLs on chromosomes 2 and 4 as well as new evidence suggesting a blood pressure QTL on chromosome 19. We have also found that rat chromosome 17 contains a QTL that influences cardiac mass independent of blood pressure. These studies demonstrate that the HXB and BXH strains can be effectively used for genome scanning studies of complex traits and have revealed new chromosome regions that may be involved in the genetic control of blood pressure and cardiac mass in rodents.

\section{Methods}

Derivation of recombinant inbred strains. The RI strains were derived from spontaneously hypertensive rats (SHR/Ola, referred to as SHR) 
and normotensive Brown-Norway rats (BN.1x/Cub, referred to as BN) (10). The BN progenitor is a BN congenic strain that carries a segment of chromosome 8 from the polydactylous PD/Cub strain (13) and as a consequence, this cross exhibits polymorphisms on chromosome 8 that are not always observed when comparing other strains of SHR and BN rats. A total of $36 \mathrm{RI}$ strains were originally derived from crosses of female SHR and male BN rats (HXB strains, $n=26$ ) or female BN rats and male SHR (BXH strains, $n=10$ ). Of the 36 original strains, 32 surviving strains are still available (22 $\mathrm{HXB}$ strains and $10 \mathrm{BXH}$ strains) and are currently beyond F35. DNA samples were available from all 36 of the original strains for analysis of the strain distribution patterns of the genetic markers.

Determination of blood pressure and cardiac mass. Blood pressure data are available for 32 of the RI strains. The blood pressures were determined by averaging direct measurements of carotid arterial pressures in five or six males $(15+/-1 \mathrm{wk}$ of age ) from each strain and have been previously reported (10). The mean arterial pressures of the strains range from $\sim 110 \mathrm{mmHg}$ to $160 \mathrm{~mm} \mathrm{Hg}$. The relative left ventricular heart weights of the RI strains were determined by averaging measurements of left ventricular heart weight corrected for body weight in the same rats used for blood pressure measurements. These data on cardiovascular phenotypes were previously obtained in collaboration with Dr. J. Kunes, Institute of Physiology, Czech Academy of Sciences, Prague (10). All rats were maintained under standard laboratory conditions and provided ad libitum access to tap water and a pelleted diet that contains $170 \mathrm{mmol} \mathrm{NaCl} / \mathrm{kg}$ (DOS 2b, VELAZ, Czech Republic).

Genetic markers and nomenclature. We analyzed the RI strain distribution patterns (SDPs) for a total of 500 biochemical, morphologic, immunogenetic, and molecular genetic markers. Typing methods and SDPs for some of these markers have been previously described (14-20).

The molecular markers used in the current study consisted primarily of microsatellite polymorphisms typeable by polymerase chain reaction analysis and microsatellite/minisatellite markers typeable by Southern blot/DNA fingerprint analysis. The PCR primers for typing the microsatellite markers of Jacob et al. (21) were obtained from Research Genetics (Huntsville, A1). PCR primers for typing other microsatellites were synthesized in the UCSF Biomolecular Resource Center from previously published sequences of Serikawa et al. (22), Goldmuntz et al. (23), Zha et al. (24), Remmers et al. (25), Kobayashi et al. (26), Deng and Rapp (27), Cicila et al. (28), and Deng et al. (29).

Multiple DNA polymorphisms were detected by Southern blot analysis of anonymous mini- and microsatellite loci using probes for synthetic tandem repeats (STRs) or natural tandem repeats (NTRs). These include over 200 new genetic markers as well as some markers that have been previously tested in the RI strains $(14,17)$. The STRs that mimic minisatellite structure can detect many polymorphic minisatellites simultaneously on Southern blots and can also be used to screen genomic libraries to identify new natural tandem repeats (30). One set of 18 minisatellite markers was isolated as previously described (31) by screening $\sim 20,000$ clones from a Sprague-Dawley rat genomic library (CLONTECH RL1032m) with the following STR probes: 14c3, 14c5, 14c14(32), 16c2, 16c4, 16c17, 16c20(33), 13c1, 14c31(34), 14c16, $16 \mathrm{c} 27$ (35). Two STR probes, (14c16 and $16 \mathrm{c} 27)$ gave a polymorphic pattern of hybridization on Southern blots. A second set of 64 anonymous minisatellite markers was obtained by another procedure to be described in detail elsewhere (manuscript in preparation). A third set of markers (NTR markers) was isolated from a rat library of short DNA fragments after screening with STR probes. Sequence analysis of these clones provided both PCR typeable markers and, after PCR amplification and purification of the repeated element, probes for use on Southern blots. Southern blots and hybridizations of the minisatellite probes were performed as previously described (32).

PCR analysis or Southern analysis was also used to detect specific gene polymorphisms not previously described between SHR and BN rats (in the genes for C-jun, Bcl2, and Nhe3). Primers amplifying a 3' portion of the rat $\mathrm{C}$-jun gene were designed from the Genbank sequence (accession number X17215): upstream primer: 5 '-aat gtg ctg gag tgg gaa gg, downstream primer: $5^{\prime}-\operatorname{tgg}$ aaa atc ttc agt gtg $\operatorname{cgg} \mathrm{c}$. Restriction fragment length polymorphism between BN and SHR rats was detected by cutting the PCR products with the Hinf I enzyme; the SHR DNA contained a Hinf I restriction site, the BN DNA did not. Primers amplifying a CA repeat microsatellite polymorphism in the $3^{\prime}$ portion of the rat $B c l 2$ gene were designed from the Genbank sequence (accession number L14680): upstream primer: $5^{\prime}$-atg aaa agg ttc act aaa gc, downstream primer: $5^{\prime}$-ata gct gat ttg acc att tgc c. PCR reactions were carried out using standard conditions or conditions described for specific primer sets $(21,22)$. The Nhe 3 gene (sodium-hydrogen exchanger 3 ) was previously assigned to chromosome 1 by somatic cell hybrid analysis (36) and in the RI strains, was detected on Southern blot analysis by cDNA probing of TaqI-digested genomic DNA which yielded a 2.7$\mathrm{kb}$ fragment in the SHR strain and a 2.9-kb fragment in the BN strain.

Determination of strain distribution patterns. Genotype results were independently scored by 2 individuals and the strain distribution patterns of the genetic markers were entered into the Map Manager computer program of Manly (version 2.5b3) (37). Data entry was verified by two individuals. The Map Manager program was used to derive a linkage map that covered $\sim 1,139$ centiMorgans $(\mathrm{cM})$ of the rodent genome. Linkage groups were assigned to specific chromosomes based on the results of somatic cell hybrid analysis, previously published chromosome locations of selected markers, or the chromosome locations of microsatellite markers reported by Jacob et al. (21). Details of the linkage map will be described in detail elsewhere (manuscript in preparation).

QTL analysis. To test for markers associated with blood pressure or cardiac weight, we used the approach that Plomin and others have used to analyze RI strains with respect to the genetics of complex phenotypes such as behavior (7-9). The method involves the use of Pearson product-moment correlation analysis in which the genetic marker information is correlated with the quantitative phenotype. Significant correlations suggest associations between markers and the quantitative trait, and also index the strength of the associations.

Because the genome scanning approach involves the testing of multiple markers, it is necessary to employ stringent statistical criteria in the linkage analysis. Neumann and Belknap have suggested statistical guidelines for genome scanning analysis of RI strains that are based on a Bayesian approach or on the Bonferroni correction for multiple comparisons $(12,38)$. For the Bonferroni adjustment, the number of independent tests is considered to be a function of the genomic distance swept by a given set of RI strains. In a set of 32 RI strains, a Bonferroni adjusted $P$ value $<.0003$ corresponds approximately to a type I error of $<.05(12,38)$. With the Bayesian approach, a $P$ value of $<.0003$ corresponds approximately to a $95 \%$ posterior probability of linkage (12). Accordingly, in the RI strain analysis of relative heart weight for which little a priori mapping information is available, we have defined statistical significance as $P<.0003$. With respect to blood pressure, however, a considerable number of studies have been published that indicate the presence of blood pressure QTLs on multiple rat chromosomes and therefore, a statistical cutoff of $P<.0003$ is excessively stringent. Thus, instead of using a strict cutoff to define statistical significance in the blood pressures studies, we have identified the genetic markers that yielded the lowest $P$ values in the Pearson correlation analyses.

\section{Results}

In the 36 recombinant inbred strains for which DNA was available, we determined the strain distribution patterns (SDPs) of 500 biochemical, immunogenetic, and molecular genetic markers. Of the 500 genetic markers tested, 453 could be assigned to specific chromosomes. The median number of markers tested per chromosome was 20 and the linked markers spanned $\sim 1,139 \mathrm{cM}$ of the genome (calculated according to the method of Jacob et al. [21]).

QTL analysis of relative left ventricular heart weight and 
A

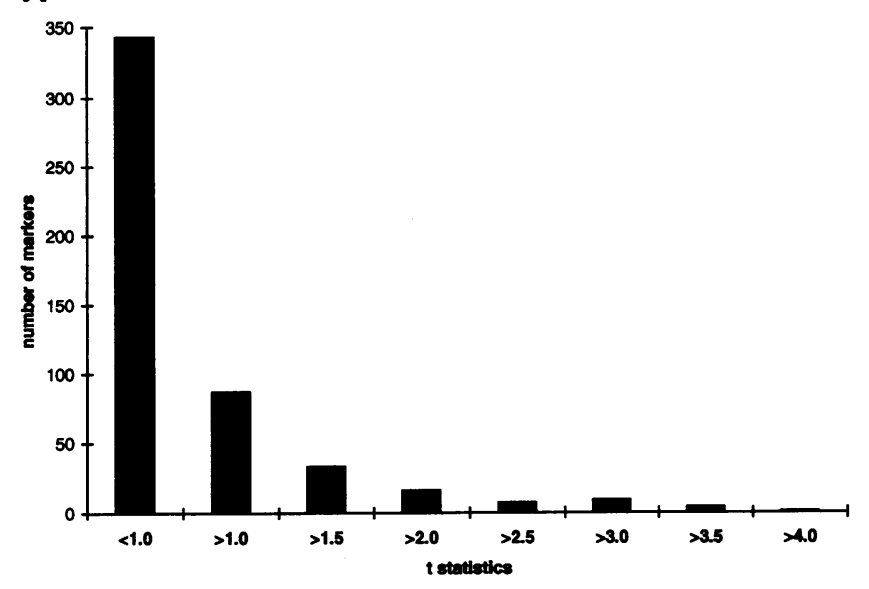

B

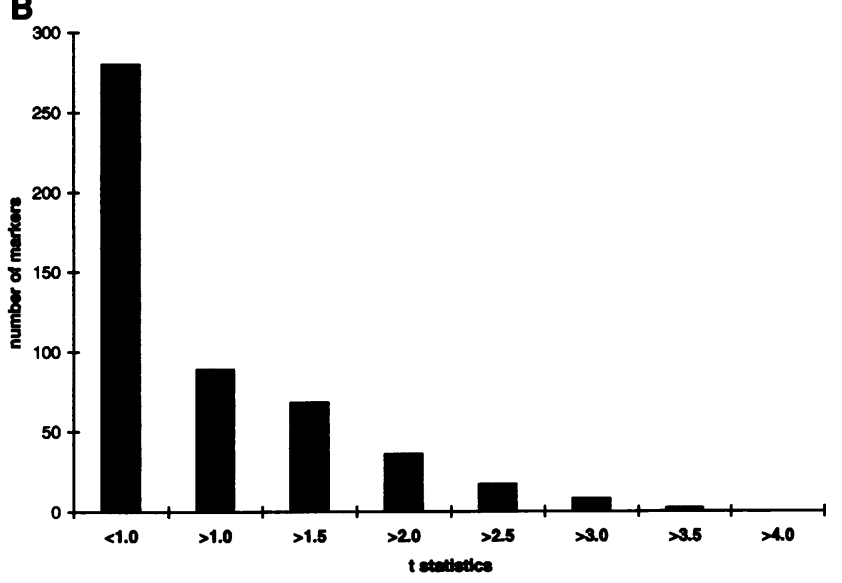

Figure 1. (A) Frequency distribution of $t$ statistics from the Pearson correlation analysis of left ventricular heart weight. A single marker, Drdla on chromosome 17 , yielded a $t$ statistic $>4(P=.00038)$. (B) Frequency distribution of $t$ statistics from the Pearson correlation analysis of systolic blood pressure. The D19Mit7 marker on chromosome 19 yielded the highest $t$ statistic in the correlation analysis of systolic blood pressure $(t=3.592, P=.0012)$. Similar frequency distributions were obtained for the analyses of diastolic and mean arterial pressures (not shown). Individual significance values for the markers showing the strongest correlations with systolic, diastolic, and mean arterial pressure are depicted in Fig. 2.

blood pressure. Fig. $1 A$ shows the frequency distribution of the $t$ statistics from the Pearson correlation analysis of the genetic markers with left ventricular heart weight (corrected for body weight). The distribution was skewed toward the lower range and the vast majority of markers yielded $t$ statistics of less than 1.0; only two markers yielded $t$ statistics $>3.6$. The genetic marker for the dopamine 1A receptor (Drdla) on chromosome 17 showed the strongest correlation with left ventricular heart weight $(t=4.0002, P=.00038, r=-0.59)$ and was the only marker that approached the level of statistical significance suggested by Neumann (12). Inheritance of the Drdla allele from the SHR progenitor was associated with lower left ventricular mass than inheritance of the Drdla allele from the BN progenitor. In a multiple linear regression using relative left ventricular heart weight as the dependent variable, and blood pressure and Drdla genotype as the independent variables ( $n$ = $32 \mathrm{RI}$ strains in the analysis), the effect of Drdla genotype on heart weight was independent of systolic, diastolic or mean arterial pressure $(P<.005$ for Drdla genotype); the effects of blood pressure on relative left ventricular heart weight in the multiple regression were not statistically significant (all $P$ $>.05$ ). The segment of chromosome 17 that contains the Drdla marker along with the significance values from linked markers on the same chromosome segment is shown in Fig. 2.

Fig. $1 B$ shows the frequency distribution of the $t$ statistics from the Pearson correlation analysis of the genetic markers with systolic blood pressure. Similar frequency distributions were obtained for the analyses of diastolic and mean arterial pressures (not shown). The marker showing the strongest correlation with systolic blood pressure was D19Mit7 on chromosome 19 ( $t=3.592, P=.0012, r=.55)$ (Fig. 2). The marker showing the strongest correlation with diastolic pressure was $D 2 N 35$ on chromosome $2(t=3.721, P=.0008, r=.56)$ (Fig. $2)$. The marker showing the strongest correlation with mean arterial pressure was $I l 6$ on chromosome $4(t=3$. 436, $P$ $=.0018, r=.53$ ) (Fig. 2). Fig. 2 shows the segments of chromosomes 2, 4, and 19 that contain these markers along with the significance values from the correlation analyses of closely linked markers on the same chromosomes.

Table I shows the mean values for systolic, diastolic, and mean arterial pressures stratified according to genotypic class (SHR versus BN) at each of the marker loci most strongly correlated with systolic, diastolic, or mean arterial pressure (D19Mit7, D2N35, and Il6). Each marker correlated with all three measures of blood pressure. In each case, inheritance of the SHR allele was associated with greater systolic, diastolic, and mean arterial pressure than inheritance of the BN allele.

\section{Discussion}

In a large set of recombinant inbred strains derived from the spontaneously hypertensive rat, we have analyzed the strain distribution patterns of $\mathbf{5 0 0}$ genetic markers to search for chromosome regions that may contain quantitative trait loci (QTLs) regulating blood pressure and cardiac mass in the rat. Of the 500 markers tested, 453 were located on specific chromosomes and spanned $\sim 1,139 \mathrm{cM}$ of the rodent genome. The results of a genome scanning analysis with these markers suggest that blood pressure regulatory loci exist on rat chromosomes 2,4 , and 19. A preliminary report of a linkage study in an $\mathrm{F} 2$ population derived from SHR and BN rats has also indicated that QTLs regulating blood pressure may exist on chromosomes 2 and 4 (39). The D2N35 marker that shows a strong correlation with diastolic blood pressure maps just distal to a marker on chromosome 2 ( $R 5159$ now designated $D 2 M g h 8)$ that Trolliet et al. reported to be linked to blood pressure in a study in saltloaded F2 rats derived from SHR and BN progenitors (39). In crosses of Dahl S rats with the WKY or MNS strains, Deng et al. have also found evidence of a blood pressure QTL in a broad region of chromosome 2 that borders the D2N35 locus (40). Given the relatively wide confidence intervals for the locations of the markers mapped in the RI strains and the fact that just a few errors in phenotyping or genotyping can substantially alter mapping results, it is quite possible that the chromosome 2 QTL detected in the RI strains is the same as that detected in the F2 crosses of Trolliet et al. and Deng et al. The Il6 marker that shows a strong correlation with mean arterial pressure borders a segment of chromosome 4 that has also been implicated 


\section{Chromosome 17 markers \\ - correlation with LVM}

\section{Chromosome 19 markers \\ - correlation with SBP}
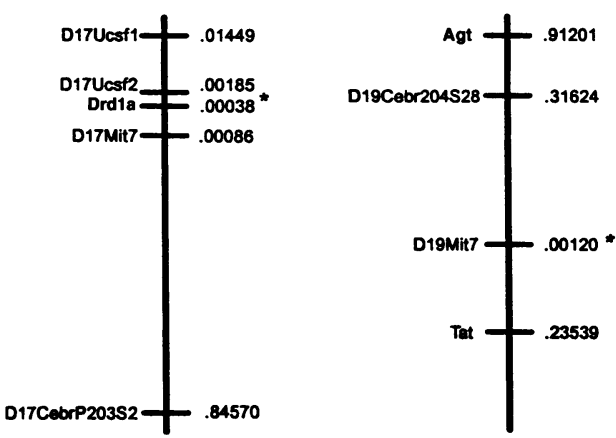

Chromosome 2 markers

- correlation with DBP

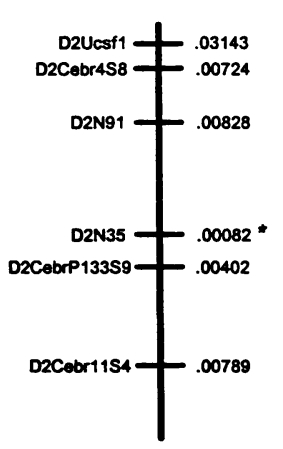

Chromosome 4 markers

- correlation with MAP

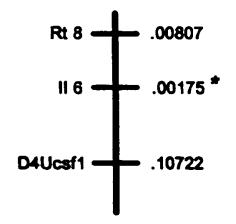

Figure 2. Chromosome segments containing the markers showing the strongest correlations with LVM (left ventricular mass), SBP (systolic blood pressure), DBP (diastolic blood pressure), and MAP (mean arterial pressure). The $P$ values from the Pearson correlation analyses are listed opposite from each marker. * The marker showing the lowest $P$ value in each correlation analysis.

in a preliminary linkage study of $\mathrm{F} 2$ rats derived from SHR and BN progenitors (39).

As emphasized by Deng et al., more precise genetic mapping of putative QTLs will require construction of congenic strains that carry different overlapping segments of the chromosome regions of interest (40). Accordingly, the current results and those from the linkage studies in $\mathrm{F} 2$ populations have motivated us to begin constructing congenic strains of SHR that carry large segments of chromosomes 2 or 4 from the Brown Norway rat. These strains are currently in the $\mathrm{N} 7$ generation and will be used to confirm the presence of the putative QTLs and to map them to more restricted chromosome regions.

The finding of a correlation between D19Mit7 and systolic blood pressure raises the possibility that a blood pressure QTL also exists on rat chromosome 19 . However, the strength of the correlation is relatively weak and little prior evidence exists suggesting that this region of chromosome 19 is involved in the genetic control of blood pressure in rats. Nevertheless, it is interesting to note that $\mathrm{D} 19 \mathrm{Mit} 7$ is linked to the angiotensinogen gene on rat chromosome 19 and this segment of chromosome 19 may be homologous to a section of human chromosome $1(41,42)$; in humans, linkage and association studies have suggested that a blood pressure QTL exists in or near the angiotensinogen gene on chromosome $1(43,44)$.

Although systolic, diastolic, and mean arterial pressures are typically well-correlated, the physiologic determinants of sys-

Table I. Marker Effects on Systolic, Diastolic, and Mean Arterial Pressure

\begin{tabular}{llccc}
\hline Marker & $\begin{array}{c}\text { Genotypic } \\
\text { class }\end{array}$ & Systolic pressure & Diastolic pressure & Mean pressure \\
\hline D19Mit7 & SHR & $164 \pm 4$ & $114 \pm 3$ & $138 \pm 3$ \\
& BN & $149 \pm 2^{\ddagger}$ & $105 \pm 2^{*}$ & $126 \pm 2^{\ddagger}$ \\
$D 2 N 35$ & SHR & $160 \pm 2$ & $115 \pm 2$ & $137 \pm 2$ \\
& BN & $151 \pm 4^{*}$ & $103 \pm 2^{\ddagger}$ & $127 \pm 3^{*}$ \\
$I l 6$ & SHR & $161 \pm 3$ & $112 \pm 2$ & $136 \pm 2$ \\
& BN & $144 \pm 3^{\ddagger}$ & $102 \pm 2^{\ddagger}$ & $122 \pm 3^{\ddagger}$ \\
\hline
\end{tabular}

Asterisks denote significance of Pearson correlations between marker genotypes and blood pressure: ${ }^{*} P<0.05 ;{ }^{\ddagger} P<0.005$. tolic pressure are not identical to those of diastolic pressure or mean arterial pressure. Accordingly, different loci might be involved in the genetic regulation of different components of the arterial pressure wave form. In the current study, however, the markers showing the strongest individual correlations with systolic, diastolic, or mean pressure showed apparent correlations with all three pressure measurements. For example, D2N35 was strongly correlated with diastolic pressure but it was also correlated, albeit less prominently, with systolic pressure and mean arterial pressure. The Il6 marker showed the strongest correlation with mean arterial pressure but it also showed correlations with systolic and diastolic pressure. It is unclear whether variation in the strength of these correlations is biologically meaningful or whether it simply represents technical error or variability in phenotyping or genotyping.

In the current study, we have also used a genome scanning approach to search for chromosome regions that may contain QTLs regulating left ventricular mass. The Drdla marker on rat chromosome 17 showed the strongest correlation with left ventricular heart weight adjusted for body weight. The results of multiple linear regression analysis indicate that the relationship between Drdla genotype and left ventricular mass is independent of blood pressure. Although the left ventricular mass of the SHR progenitor strain is greater than that of the $\mathrm{BN}$ progenitor, the mean left ventricular mass of the RI strains that inherited the Drdla allele of the SHR progenitor was less than that of the RI strains that inherited the Drdla allele of the BN progenitor. These findings demonstrate that the SHR may have fixed at least one allele that promotes lower left ventricular mass than the corresponding allele fixed by the BN progenitor.

Although previous studies have indicated that genetic factors are important determinants of cardiac size in the rat, few attempts have been made to map specific chromosome regions that contain QTLs contributing to normal variation in cardiac mass $(45,46)$. Dopamine receptors are clearly of functional significance in myocardial tissue, and it is tempting to speculate about Drdla gene polymorphisms and ventricular growth. However, these kinds of linkage studies can only identify relatively broad chromosome regions that may contain QTLs regulating complex phenotypes and therefore, attempts to draw conclusions about the functional role of specific variants in those regions should be regarded with caution. The possibility that 
chromosome 17 contains a QTL regulating cardiac mass can now be directly pursued by comparing left ventricular heart weights in congenic strains of SHR that are genetically identical except for selected segments of this chromosome.

In the current study, we determined the strain distribution patterns of $>230$ new anonymous markers derived from a variety of minisatellites that yield mono-locus or multi-locus patterns on Southern blot analysis. Although these newly typed anonymous markers are not particularly convenient for use in genetic studies in segregating populations, they represent a very useful addition to the cumulative RI strain marker set and should facilitate genome scanning and mapping studies in the HXB and BXH strains The new minisatellite markers were found to be dispersed throughout the rat genome and appeared to be distributed equally among chromosomes according to chromosome size (with the possible exception of the $\mathrm{X}$ chromosome for which a relative deficiency of repetitive sequence markers has also been noted by Dietrich et al. in the mouse [47] and Jacob et al. in the rat [21]).

Genome scanning approaches have not been widely used to study the pathogenesis of cardiovascular disorders or other complex phenotypes in the rat. The limited use of genome scanning approaches for mapping QTLs regulating complex phenotypes can be attributed in part to the substantial amount of time and labor required to genotype hundreds of $\mathrm{F} 2$ or backcross animals for multiple loci on every chromosome. Based on the results of the current study, we now have a large set of extensively genotyped rat RI strains that can be efficiently used for the preliminary genetic dissection of a variety of complex phenotypes. Genome scanning studies in these strains implicate chromosomes 2, 4 and possibly 19 in the genetic control of blood pressure, and chromosome 17 in the genetic control of left ventricular mass. In addition to providing an efficient screening tool for mapping studies, these RI strains will also be useful for studying environment-genotype interaction in the control of physiologic traits. In contrast to the finite life spans of individual genotypes represented in F2 or backcross populations, the genotypes represented among recombinant inbred strains are perpetually available and can be repeatedly studied. Thus, the RI strains can be used for studies of QTL action in a range of different environments and with different experimental techniques. These features, together with the cumulative nature of RI strain marker sets should greatly facilitate the use of combined genetic and physiologic approaches in the study of blood pressure and other complex phenotypes in the rat.

Note added in proof. Lodwick and co-workers have also recently reported that a marker on chromosome 19 (Agt) is linked to the inherited control of blood pressure in SHR (1995. Hypertension. 25:1245-1251).

\section{Acknowledgments}

We thank Alena Musilova for technical assistance in the breeding of the RI strains, A. Panczak for providing the strain distribution pattern of the Gox alleles, G. Butcher for providing the strain distribution pattern of the Rt6 alleles, and J. Orlowski for the gift of the Nhe 3 cDNA probe.

This work was supported by grant NO Z-548-3 from the Ministry of Health of the Czech Republic to VK, M Pravenec, and V Bila; a grant from the US-Czechoslovak Science and Technology Program to VK, M Printz, M Pravenec, and TK; and grants from the National Heart, Lung, and Blood Institute to TK, MAS, and M Printz, the EEC
EUROGEM project and the GIP-GREG to GV, and the FRSM and the Association contre le Cancer to CS and JS.

\section{References}

1. Lander, E. S., and D. Botstein. 1989. Mapping Mendelian factors underlying quantitative traits using RFLP linkage maps. Genetics. 121:185-199.

2. Taylor, B. A. 1978. Recombinant inbred strains: use in gene mapping. In Origins of Inbred Mice. H. C. Morse III, editor. Academic Press, New York. 423-438.

3. Taylor, B. A., A. Navin, and S. J. Phillips. 1994. PCR-amplification of simple sequence repeat variants from pooled DNA samples for rapidly mapping new mutations of the mouse. Genomics. 21:626-632.

4. Taylor, B. A., and P. C. Reifsnyder. 1993. Typing recombinant inbred mouse strains for microsatellite markers. Mamm. Genome. 4:239-242.

5. Jeffreys, A. J., V. Wilson, R. Kelly, B. Taylor, and G. Bulfield. 1987. Mouse DNA fingerprints: analysis of chromosome localization and germ-line stability of hypervariable loci in recombinant inbred strains. Nucleic Acids Res. 15:2823-2836.

6. St.Lezin, E. M., M. Pravenec, and T. W. Kurtz. 1993. New genetic models for hypertension research. Trends Cardiovasc. Med. 3:119-123.

7. Crabbe, J. C., J. K. Belknap, and K. J. Buck. 1994. Genetic animal models of alcohol and drug abuse. Science (Wash. DC). 264:1715-1723.

8. Plomin, R., G. E. McClearn, G. Gora-Maslak, and J. M. Neiderhiser. 1991. Use of recombinant inbred strains to detect quantitative trait loci associated with behavior. Behav. Genet 21:99-116.

9. Gora-Maslak, G., G. E. McClearn, J. C. Crabbe, T. J. Phillips, J. K. Belknap, and R. Plomin. 1991. Use of recombinant inbred strains to identify quantitative trait loci in psychopharmacology. Psychopharmacology (Berl.). 104:413-424.

10. Pravenec, M., P. Klir, V. Kren, J. Zicha, and J. Kunes. 1989. An analysis of spontaneous hypertension in spontaneously hypertensive rats by means of new recombinant inbred strains. J. Hypertension. 7:217-222.

11. Neumann, P. E., and R. L. Collins. 1991. Genetic dissection of susceptibility to audiogenic seizures in inbred mice. Proc. Natl. Acad. Sci. USA. 88:54085412.

12. Neumann, P. E. 1992. Inference in linkage analysis of multifactorial traits using recombinant inbred strains of mice. Behav. Genet. 22:665-676.

13. Kren, V. 1975. Genetics of the polydactyly-luxate syndrome in the Norway rat, Rattus norvegicus. Acta Univ. Carol. Med. [Monogr. 68]. (Praha). 1-103.

14. Pravenec, M., V. Kren, V. Bila, P. Kabra, M. Krsiakova, L. Simonet, and T. Kurtz. 1990. HXB and BXH recombinant inbred strains: Strain distribution patterns of some genetic markers. Transplant Proc. 22:2557-2558.

15. Pravenec, M., V. Kren, J. Kunes, A. G. Scicli, O. A. Carretero, L. Simonet, and T. W. Kurtz. 1991. Cosegregation of blood pressure with a kallikrein gene family polymorphism. Hypertension. 17:242-246.

16. Hamet, P., D. Kong, M. Pravenec, J. Kunes, V. Kren, P. Klir, Y. L. Sun, and J. Tremblay. 1992. Restriction fragment length polymorphism of hsp70 gene, localized in the RT1 complex, is associated with hypertension in spontaneously hypertensive rats. Hypertension. 19:611-614.

17. Kurtz, T. W., L. Simonet, V. Kren, and M. Pravenec. 1991. Gene mapping in experimental hypertension. In Genetic Approaches to Coronary Heart Disease and Hypertension. K. Berg, V. Bulyzhenkov, Y. Christen, and P. Corvol, editors. Springer-Verlag, Heidelberg. 38-59.

18. Pravenec, M., E. M. St.Lezin, V. Kren, J.-M. Wang, and T. W. Kurtz. 1994. Linkage mapping of alkaline phosphatase 1 , inhibin $\alpha$ subunit, and gammacrystallin 1 on rat chromosome 9 and $\mathrm{Na}^{+}, \mathrm{K}^{+}$-ATPase $\alpha_{2}$ subunit, renin, and leukocyte common antigen on rat chromosome 13. Genomics. 19:190-191.

19. Kren, V., M. Pravenec, V. Bila, D. Svobodova, E. St.Lezin, and T. W. Kurtz. 1993. Linkage between the RT8 alloantigen and interleukin 6 loci on the rat chromosome 4. Transplant. Proc. 25:2777.

20. Bender, K., R. T. Siebert, T. F. Wienker, V. Kren, M. Pravenec, and S. Bissbort. 1994. Biochemical genetics of methylglyoxal dehydrogenases in the laboratory rat. Biochem. Genet. 32:147-154.

21. Jacob, H., D. Brown, R. Bunker, M. Daly, V. Dzau, A. Goodman, G. Koike, V. Kren, T. Kurtz, A. Lernmark, G. Levan, Y. Mao, A. Pettersson, M. Pravenec, J. Simon, C. Szpirer, J. Szpirer, M. Trolliet, E. Winer, and E. Lander. 1995. A genetic linkage map of the laboratory rat. (Rattus norvegious) Nat. Genet. 9:63-69.

22. Serikawa, T., T. Kuramoto, P. Hilbert, M. Mori, J. Yamada, C. J. Dubay, K. Lindpainter, D. Ganten, J. L. Guenet, G. M. Lathrop, and J. S. Beckmann. 1992. Rat gene mapping using PCR-analyzed microsatellites. Genetics. 131:701721.

23. Goldmuntz, E. A., E. F. Remmers, H. Zha, J. M. Cash, P. Mathern, L. J. Crofford, and R. L. Wilder. 1993. Genetic map of 12 polymorphic loci on rat chromosome 1. Genomics. 16:761-764.

24. Zha, H., R. L. Wilder, E. A. Goldmuntz, J. M. Cash, L. J. Crofford, P. Mathern, and E. F. Remmers. 1993. Linkage map of 10 polymorphic markers on rat chromosome 2. Cytogenet. Cell Genet. 63:117-122. 
25. Remmers, E. F., E. A. Goldmuntz, H. Zha, P. Mathern, Y. Du, L. J. Crofford, and R. L. Wilder. 1993. Linkage map of nine loci defined by polymorphic DNA markers assigned to rat chromosome 13. Genomics. 18:277-282.

26. Kobayashi, E., M. Tachibana, H. Ikadai, T. Imamichi, and T. Kunieda. 1992. Linkage among esterase-6 (Es-6), neural cell adhesion molecule (NCAM), and apolipoprotein C-III (apoc-3) genes on rat chromosome 8. Mamm. Genome. 3:656-658.

27. Deng, Y., and J. P. Rapp. 1992. Cosegregation of blood pressure with angiotensin converting enzyme and atrial natriuretic peptide receptor genes using Dahl salt-sensitive rats. Nat. Genet. 1:267-272.

28. Cicila, G. T., J. P. Rapp, J.-M. Wang, E. St.Lezin, S. C. Ng, and T. W. Kurtz. 1993. Linkage of $11 \beta$-hydroxylase mutations with altered steroid biosynthesis and blood pressure in the Dahl rat. Nature Genet. 3:346-353.

29. Deng, A. Y., H. Dene, M. Pravenec, and J. P. Rapp. 1994. Genetic mapping of two new blood pressure quantitative trait loci in the rat by genotyping endothelin system genes. J. Clin. Invest. 93:2701-2709.

30. Vergnaud, G., D. Gauguier, J. J. Schott, D. Lepetit, V. Lauthier, D. Mariat, and J. Buard. 1993. Detection, cloning, and distribution of minisatellites in some mammalian genomes. Exs. 67:47-57.

31. Vergnaud, G., D. Mariat, F. Apiou, A. Aurias, M. Lathrop, and V. Lauthier 1991. The use of synthetic tandem repeats to isolate new VNTR loci: cloning of a human hypermutable sequence. Genomics. 11:135-144.

32. Vergnaud, G. 1989. Polymers of random short oligonucleotides detect polymorphic loci in the human genome. Nucleic Acids. Res. 17:7623-7630.

33. Vergnaud, G., D. Mariat, M. Zoroastro, and V. Lauthier. 1991. Detection of single and multiple polymorphic loci by synthetic tandem repeats of short oligonucleotides. Electrophoresis. 12:134-140.

34. Mariat, D., and G. Vergnaud. 1992. Detection of polymorphic loci in complex genomes with synthetic tandem repeats. Genomics. 12:454-458.

35. Mariat, D., B. De Gouyon, C. Julier, M. Lathrop, and G. Vergnaud. 1993. Genetic mapping through the use of synthetic tandem repeats in the mouse genome. Mamm. Genome. 4:135-140.

36. Szpirer, C., J. Szpirer, M. Riviere, G. Levan, and J. Orlowski. 1994. Chromosomal assignment of four genes encoding $\mathrm{Na} / \mathrm{H}$ exchanger isoforms in human and rat. Mamm. Genome. 5:153-928.
37. Manly, K. F. 1993. A Macintosh program for storage and analysis of experimental genetic mapping data. Mamm. Genome. 4:303-313.

38. Belknap, J. K. 1992. Empirical estimates of Bonferroni corrections for use in chromosome mapping studies with the BXD recombinant inbred strains. Behav. Genet. 22:677-684.

39. Trolliet, E. M., J. E. Krieger, N. J. Schork, G. Koike, K. G. Franchini, J. S. Simon, E. M. Krieger, E. S. Lander, V. J. Dzau, and H. J. Jacob. 1994 Systolic blood pressure after salt loading is caused by 4 to 6 quantitative trait loci acting in an additive fashion. Hypertension. 24:387 (Abstr.)

40. Deng, A. Y., H. Dene, and J. P. Rapp. 1994. Mapping of a quantitative trait locus for blood pressure on rat chromosome 2. J. Clin. Invest. 94:431-436.

41. Mori, M., K. Ishizaki, T. Yamada, H. Chen, T. Sugiyama, T. Serikawa and J. Yamada. 1989. Restriction fragment length polymorphisms of the angiotensinogen gene in inbred rat strains and mapping of the gene on chromosome 19q Cytogenet. Cell Genet. 50:42-45.

42. Yamada, J., T. Kuramoto, and T. Serikawa. 1994. A rat genetic linkage map and comparative maps for mouse or human homologous rat genes. Mammalian Genome. 5:63-83.

43. Jeunemaitre, X., F. Soubrier, Y. V. Kotelevtsev, R. P. Lifton, C. S. Williams, A. Charru, S. C. Hunt, P. N. Hopkins, R. R. Williams, J. M. Lalouel, and P. Corvol. 1992. Molecular basis of human hypertension: Role of angiotensinogen. Cell. 71:169-180.

44. Caulfield, M., P. Lavender, M. Farrall, P. Munroe, M. Lawson, P. Turner, and A. J. J. Clark. 1994. Linkage of the angiotensinogen gene to essential hypertension. N. Engl. J. Med. 330:1629-1633.

45. Tanase, H., Y. Yamori, C. T. Hansen, and W. Lovenberg. 1982. Heart size in inbred strains of rats. Hypertension. 4:864-872.

46. Kunes, J., V. Kren, P. Klir, J. Zicha, and M. Pravenec. 1990. Genetic determination of heart and kidney weights studied using a set of recombinan inbred strains: the relationship to blood pressure. J. Hypertens. 8:1091-1095.

47. Dietrich, W. F., J. C. Miller, F. G. Steen, M. Merchant, D. Damron, R. Nahf, A. Gross, D. C. Joyce, M. Wessel, R. D. Dredge, A. Marquis, L. D. Stein N. Goodman, D. C. Page, and E. S. Lander. 1994. A genetic map of the mouse with 4,006 simple sequence length polymorphisms. Nature Genet. 7 Suppl. 220 245. 